\title{
Model for improving the accuracy of relevant project selection in analogy using differential evolution algorithm
}

\author{
I THAMARAI $^{1, *}$ (D) and S MURUGAVALLI ${ }^{2}$ \\ ${ }^{1}$ Sathyabama University, Chennai 600119, India \\ ${ }^{2}$ Panimalar Engineering College, Chennai 600123, India \\ e-mail: ilango.thamarai@gmail.com; murugavalli26@rediffmail.com
}

MS received 23 March 2015; revised 27 April 2016; accepted 28 August 2016

\begin{abstract}
Software effort estimation is the process of calculating the effort required to develop a software product based on the input parameters that are usually partial in nature. It is an important task but the most difficult and complicated step in the software product development. Estimation requires detailed information about project scope, process requirements and resources available. Inaccurate estimation leads to financial loss and delay in the projects. Due to the intangible nature of software, most of the software estimation process is unreliable. But there is a strong relationship between effort estimation and project management activities. Various methodologies have been employed to improve the procedure of software estimation. This paper reviews journal articles on software development to get the direction in the future estimation research. Several methods for software effort estimation are discussed in this paper, including the data sets widely used and metrics used for evaluation. The use of evolutionary computational tools in the estimation is dealt with in detail. A new model for estimation using differential evolution algorithm called DEAPS is proposed and its advantages are discussed.
\end{abstract}

Keywords. Software effort estimation methods; algorithmic and non-algorithmic models; evolutionary computation; differential evolution.

\section{Introduction}

Planning a software project is one of the most important activities in any software development process. Many factors are to be considered to estimate the software cost and effort. The most important factors are size of the project, number of persons involved and schedule. Prediction of software effort is a difficult and complicated task. Software is intangible in nature and so the measurement of progress in the software process is very difficult to access. Also the requirements of a software project change continually, which causes changes in estimation. Inaccurate estimation of effort is the usual cause of software project failures. Jorgensen et al [1] suggested that receiving feedback about other software professional's estimation performance leads to more unrealistic assessments. Budget and schedule pressure also plays an important role in effort calculation [2]. Laird [3] lists out the reasons for the inaccurate software project estimation. They are:
a. lack of training
b. confusion in the schedule
c. inability of team members

*For correspondence d. incomplete and changing requirements

e. hope-based planning

Jorgensen and Sheppard [4] presented a systematic review from various journals. According to them, the properties of dataset impact the result when evaluating the estimation. Dejaeger et al [5] presented an overview of the literature related to software effort estimation. The parameters used were project size development and environmentrelated attributes. The paper gives a detailed study with different processing steps and addresses many issues like data quality, missing values, etc. The authors confirm that the Constructive Cost (COCOMO) model performed equally well as the non-linear techniques. Menzies et al [6] evaluated the lessons that are global to multiple projects and local to particular projects in software effort estimation. They used the Promise repository and concluded that software effort estimation team should build clusters from the available data and the best cluster is the one that is near the source data but not from the same source as the test data.

Kocaguneli et al [7] explored the general principles of effort estimation that can guide the design of effort estimation. The authors conclude that the effort estimation shall improve significantly if the situations when the assumptions are violated are identified and removed. Also it 
is said in the paper that estimation can be improved by dynamic selection of nearest neighbour with small variance. Mittas and Angelis [8] proposed a statistical framework based on multiple comparison algorithms to rank several cost estimation models. Harman and Mausouri [9] proposed the application of search-based optimization for the software effort estimation. They listed out the advantages of using search-based optimization as robustness, scalability and power. Kocaguneli et al [10] proposed a tool called QUICK TOOL to reduce the number of features and instances required to capture the information for software effort estimation. This reduces the complexity in data interpretation. The distance between features is taken for analysis. The tool is suitable for small data sets. Ashman [11] suggested a simple Use Case based model. In this, the relationship between estimated and actual data is used to improve the future estimates. This model works best in an interactive development process. The aim of the Use Case based model is to capture the experience of specialists and enable a consistency on the timescale. As today's software products are largely component based, the prediction is also done on the basis of individual components of the software. The performance of a system can be predicted by creating models to calculate the performance of every single component that comprises the full system.

To improve the uncertainty in cost assessments, Jorgensen [12] provided evidence-based guidelines. The methods for assessing software development cost uncertainty are based on results from empirical studies. Some of the important guidelines provided are as follows: not to rely solely on unaided, intuition-based uncertainty assessment process and to apply structured and explicit judgment-based process. His advice is to combine uncertainty assessments from different sources through group work and not through mechanical combination. Thus it can be seen that various aspects have to be considered for software effort estimation models. The main aim of this paper is to give a detailed description about various software effort estimation methods. This paper is organized as follows. Section 2 consists of discussion on various traditional software estimation methods and Algorithmic Models. Section 3 gives a brief description of the Non-Algorithmic Models. In section 4, we discuss about the recent trend of using evolutionary computation methods in software effort estimation. In section 5, we propose a new model called DEAPS for the selection of most relevant project in Analogy for Effort Estimation and its experimental results. Section 6 is the conclusion and has recommendation for future work.

\section{Software estimation methods}

There are many traditional methods such as the Expert Judgment method, Function Point (FP) method, COCOMO, SLIM Model (Software Life Cycle Management model) and Case-Based Reasoning (CBR) Model to the recent methods that use Neural Networks, Fuzzy Logic, Genetic Algorithm (GA), Genetic Programming (GP), Particle Swarm Optimization, etc. Figure 1 shows the classification of different effort estimation methods.

The software effort estimation models are primarily divided into four main categories. The first and foremost is the Expert Judgment Method, where the effort is estimated by experts in the field. Algorithmic models are based on the mathematical formulas. Some of the Algorithmic Models are Function Point (FP), Constructive Cost Model (COCOMO) and Software Life Cycle Management model (SLIM). These models depend on various parameters like LOC (Lines of code), Complexity, Number of Interfaces, etc. The limitation of Algorithmic models such as inflexibility led to the Non-algorithmic models. Case-Based Reasoning (CBR) is a popular Non-algorithmic method. Analogy is a CBR methodology. Also with the advent of soft computing techniques, new methodology of Evolutionary computation came into existence. Many Machine Learning methods are used, including Neural Networks, GA, GP and DE. These methods are discussed in the following sections.

All the approaches have their own advantages and disadvantages. It should be noted that there is not a single method that can be said to be the best for all situations. Some of the research favors the combination of methods that has been proved successful. Hsu et al [13] integrated several software estimation methods and assigned linear weights for combinations. They proved that their model is very useful in improving estimation accuracy. Jorgensen and Grimstad [14] made a detailed study on how misleading and irrelevant information can affect software estimation. They have presented the research questions and hypothesis. Based on the answers, they concluded that the field settings that led to irrelevant information have very small impact on the effort estimation than the artificial experimental settings. They also summarized that the researchers should be more aware of the different roles of lab and field experiments.

\subsection{Expert judgment method}

This was the first method used to estimate the software development effort. In this, one or more experts give the estimation based on their experience in the field after analysing the problem thoroughly. The popular example for this method is Delphi Estimation Method, in which many experts give an independent estimate. The mean of these values is taken as the effort needed. The Standard Delphi technique was later replaced by Wideband Delphi technique.

Jorgensen [15] presented a detailed review on expert estimation of software development effort. He evaluates 12 best practice guidelines in the expert estimation in this review paper. They are: (1) Avoiding irrelevant and 


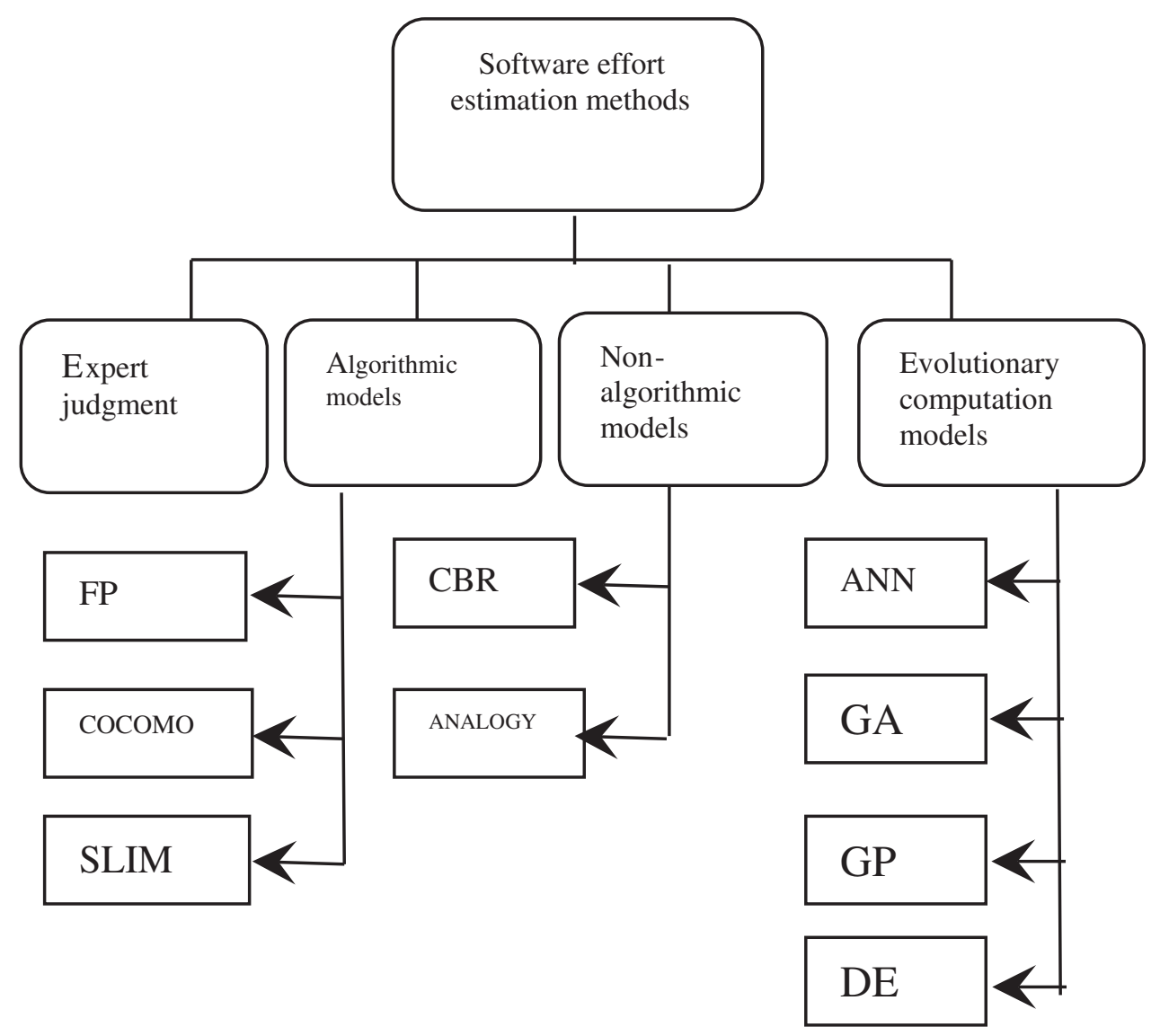

Figure 1. Classification of software estimation models.

unreliable estimation information, (2) Asking the estimators to criticize their method of estimation, (3) Avoiding conflicts in estimation, (4) Estimating but avoiding pressure, (5) Using the past data, (6) Choosing the expert from the relevant field, (7) Combining different expert's estimation values, (8) Accessing the uncertainties, (9) Estimating independently, (10) Using checklists, (11) Providing proper training in estimation and (12) Providing feedback on accuracy in estimation. He also emphasizes the evidence to support the 12 principles. The author concludes by saying that expert estimation is a very powerful strategy but the use of model can certainly reduce the human biases. The main advantage of the Expert Judgment Method is that it is a cheap and effective method. The disadvantage is that it is always a subjective method and cannot be proved.

\subsection{Algorithmic models}

Estimation is based on the mathematical models that calculate effort as a function of a number of variables. It takes the form

$$
\text { Effort }=f\left(x_{1}, x_{2}, \ldots, x_{\mathrm{n}}\right)
$$

where $\left(x_{1}, x_{2}, \ldots\right)$ are called cost factors which can be Product attributes, Hardware attributes, Personnel attributes and Project attributes. The most commonly used Algorithmic Models are FP, SLIM and COCOMO.

2.2a Function point (FP): The FP Evaluation Method was proposed by Albrecht. The idea of the FP is that the size of a software product depends on the number of functions it supports. FP is computed in two steps. The first step is to compute the unadjusted function point (UFP). The second step is to compute the technical complexity factor (TCF)

\section{Step 1: Calculation of UFP}

UFP $=$ (Number of inputs) $\times 4+$ (Number of outputs) $\times 5+$ (Number of inquires) $\times 4+$ (Number of files) $\times 10+$ (Number of interfaces) $\times 10$

Step 2: Calculation of TCF

This depends on 14 factors such as transaction rate, reusability, data communications, backup and recovery, and performance.

$$
\mathrm{TCF}=0.65+(\text { Sum of Factors }) / 100
$$

Based on the calculation of UFP and TCF, FP is calculated: 


$$
\mathrm{FP}=\mathrm{UFP} \times \mathrm{TCF}
$$

FP analysis helps the Project Management people to monitor the project. This is because, as new functions are removed or changed during the project, the FP count is updated. Hence the scope of the project can be tracked easily. The main disadvantage of FP metric is that it does not take into account the complexity in the algorithm of the software

2.2b Constructive cost model (COCOMO): The COCOMO model was proposed by Barry Boehm. This model estimates the effort by dividing the project into three categories based on the size, as Organic, Semi-detached and Embedded. There are three types of COCOMO models namely Basic Model, Intermediate Model and Complete Model. In the Basic Model, the calculation of effort is based on the project size $(L)$ and the equations are given below:

Organic Mode $=2.4 L^{1.05}$

Semi-detached Mode $=3.0 L^{1.12}$

Embedded Mode $=3.6 L^{1.20}$

In the Intermediate COCOMO, the estimation of effort is calculated based on the project size and a set of 15 cost drivers or attributes. Each of the 15 attributes gets a rating value. The product of these values is called the Effort Adjustment Factor (EAF). The equations for effort calculation are given below:

Organic mode $=3.2 L^{1.05} \times \mathrm{EAF}$

Semi-detached Mode $=3.0 L^{1.12} \times$ EAF

Embedded mode $=2.8 L^{1.20} \times$ EAF

In the complete COCOMO model, the effort is calculated for each step of the software development cycle. Individual results in each step are summed up to get the total effort. The third approach decreases the error. In recent years, the use of COCOMO model has largely reduced as it is difficult to use this model in the multiple platforms. The COCOMO model is easy to interpret. It considers the factors that affect the effort of the project. The demerit is that it ignores requirements and documentation. Menzies et al [16] proposed the COSEEKMO model to avoid the large deviation problem that may arise due to comparison of two or more best practices in estimation. COSEEKMO was designed after a detailed analysis of several causes of deviation. It is shown that MRE errors are reduced in this model. The main advantage of COSEEKMO is that the analysis is fully automatic but the input project data should be specified only in COCOMO format.

2.2c Software life cycle management (SLIM): SLIM is one of the first Algorithmic Cost Models developed by Putnam. The Formula used is

$$
\text { Effort }=\left(\mathrm{LOC} /\left(c t^{4 / 3}\right)\right) \times 3
$$

In the above formula, LOC is the size of the project, $t$ is the development time and $c$ is a technology constant that depends on the tools, languages and methodology. The main advantage is that the measure is sensitive to the technology constant. But, it is not suitable for small projects and the uncertainty in the software size may result in inaccurate estimation.

\section{Non-algorithmic models}

In these models, the estimation is done by analysing the previous projects. The most popular methodology is CaseBased Reasoning (CBR).

\subsection{Case-based reasoning (CBR)}

CBR consists of four stages:

1. Retrieval of similar cases

2. Reduction of cases that are retrieved, to find a reasonable solution

3. Revision of the solution

4. Retaining the solution for a new case

Estimation of Analogy is a CBR method. Some of the CBR-based tools that are used for cost estimation are FACE, ANGEL and ESTOR.

\subsection{Analogy-based estimation (ABE)}

The basic idea of ABE is to compare the proposed new project parameters with the previous projects to obtain the relevant project. This is then used to estimate the effort of new project. An analogous estimate is based on the past projects and so the estimate can be substantiated. Although the process is similar to human thinking, it is hard to combine the cases. Prediction largely depends on the cases that have been observed. The similarity between the testing project and historical data base is determined by similarity measure. This gives the distance between two objects in an $n$-dimensional space. The most commonly used metric is the Euclidean distance metric. The Euclidean distance between 2 points $\mathrm{P}\left(p_{1}, p_{2}, \ldots ., p_{n}\right)$ and $\mathrm{Q}\left(q_{1}, q_{2}, \ldots, q_{n}\right)$ is calculated as

$$
\begin{aligned}
& \sqrt{(p 1-q 1)^{2}+(p 2-q 2)^{2}+\cdots(p n-q n)^{2}} \\
& =\sqrt{\sum_{i=1}^{n}(p i-q i)^{2}}
\end{aligned}
$$

The weighted Euclidean distance is calculated as

$$
\begin{aligned}
& \sqrt{w 1(p 1-q 1)^{2}+w 2(p 2-q 2)^{2}+\cdots w n(p n-q n)^{2}} \\
& =\sqrt{\sum_{i=1}^{n} w i(p i-q i)^{2}}
\end{aligned}
$$


Auer et al [17] proposed allocation of separate weights to each project feature and find the optimized weights by extensive search. According to the authors, this method increases estimation accuracy and reliability. In this approach, every feature has a distinct influence on the search for similar projects in a historical database. This eliminates the need for experts to set the weights manually. Suwanjang and Prompoon [18] proposed a framework for developing a model for software cost estimation based on a relational matrix of a project profile for their study. They chose a human resource management related company, which has many software modification projects.

\section{Evolutionary computation models}

The use of Evolutionary Computation Models was suggested recently to estimate the software projects. They have the advantage of handling large search spaces. The basic idea is the Darwin's theory of evolution according to which the genetic operations between chromosomes lead to the survival of the fittest individuals. These methods are the extension of machine learning algorithm such as ANN.

\subsection{Artificial neural network (ANN)}

ANN Models are inspired by the human neural system to solve problems. An ANN is an information-processing system that has certain performance characteristics in common with the biological neural network. This type of network has two layers, namely input layer and output layer. There are links between the layers. Each link carries weights. There can be hidden layers in between these layers. Back propagation Algorithm is the most popular method for training. In this, there are two passes, a forward pass and a backward pass. In the forward pass, the weights are all fixed. During the backward pass, the weights are adjusted according to the error correction rules. The weight adjustment is based on the error produced between the desired output and actual output. Mair et al [19] conclude that Artificial Neural Networks offer accurate effort prediction but their configuration and interpretation are difficult to achieve.

Finnie and Wittig [20] examined the performance of ANN and CBR tools for software effort estimation. The concept of back propagation neural network on Desharnais data set and ASMA (Australian Software Metric Association) data set were explored by them. They proved that ANN results in high level of accuracy. They concluded that ANN models are capable of providing good estimation models. They also said that the performance depends on the training data. Malhotra and Jain [21] evaluated the use of machine learning methods such as ANN, Decision tree and Support Vector Machine in effort prediction. According to their research, it is proved that the decision tree method is the best method among the three. The advantage of Artificial Neural Networks is that they can handle heterogeneous database but there are no guidelines for design. Accuracy largely depends on the training data set.

\subsection{Genetic algorithm (GA)}

GA is a search-based algorithm to get optimal solutions. It is an evolutionary computation method. In the GA, consecutive populations of individuals are created, from which we get the optimal solution for the given problem. The search process is influenced by the following components:

a. An encoding of solutions to the problem known as chromosomes

b. A function to evaluate the fitness

c. Initialization of initial population

d. Selection operator

e. Reproduction operator

The important issues related to GA are the representation of solution, selection of genetic operators and choosing the best fitness function. GA can efficiently search through the solution space of complex problem. Li et al [22] used GA for project selection. The steps involved are encoding, population generation, fitness function evaluation, crossover, mutation, elitism and stopping criteria. The two realworld data sets Desharnais data set and Albrecht data set are used for the experiments. The authors applied two ABE-based models on these data sets. The first model uses GA to select appropriate projects subsets. This is named as PSABE (Project Selection in Analogy-Based Estimation). The second model FWABE (Feature Weighting ABE) assigns relevant feature weights by GA. The results are better than those from the other software estimation methods. Krogmaun et al [23] presented a reverse engineering approach that combines genetic search, static and dynamic analysis to predict the performance of software application. The performance of file-sharing approach is predicted using runtime byte code count. The average accuracy is proved to be better than that by other methods. The main disadvantage is that this approach supports only component for which java byte code is available. This method is useful for component-based applications.

\subsection{Genetic programming $(G P)$}

GP is a field of Evolutionary Computation that works on tree data structure. Non-continuous functions are very common in software engineering applications due to the use of branching statements. GP can be effectively used in such situations. Using a tree-based representation in GP requires adaptive individuals and domain-specific grammar. GP begins with a population of randomly created programs. The programs consist of functions corresponding to the problem domain. Each program is evaluated based on a 
fitness function. Unlike GA, mutation operation is usually not needed in GP because the crossover operation can provide point mutation at nodes. The process of selection and crossover of individuals continues till the termination criteria are satisfied. Burgess and Lefley [24] analysed the potential of GP in Software Effort Estimation in terms of accuracy and ease of use. The research was based on Desharnais data set of 81 software projects. The authors proved that the use of GP offers improvement in accuracy but this improvement depends on the measure and interpretation of data used.

\subsection{Differential evolution (DE)}

$\mathrm{DE}$ is an important evolutionary computation method in recent days that can be used to improve the exploration ability. DE is similar to GA, but it differs in the sense that distance and direction information from the current population is used to guide the search process. DE is a method that optimizes a problem iteratively to improve a solution . There are many types of DE such as Simple DE, Population-based DE and Compact DE. DE performs better than any other contemporary algorithm and it is proved that it offers good optimization due to higher number of local optima and higher dimensionality.

In a simple DE algorithm, an initial population is created by a random set of individuals. For each generation, three individuals, say $x_{1}, x_{2}$ and $x_{3}$, are selected. An off-spring $x_{\text {off }}^{\prime}$ is generated by mutation as $x_{\text {off }}^{\prime}=x_{1}+F\left(x_{2}-x_{3}\right)$. Here, $F$ is a scale factor. Then crossover is done based on some condition. In Thamarai and Murugavalli [25], a new method is proposed where a DE algorithm is used for the selection of similar projects by the analogy method. In the proposed algorithm, which is based on population-based DE, the primary population (Pp) set consists of selected individuals. The secondary population (Ps) serves as an archive of those offspring rejected by the selection operator. In compact DE, Mininno et al [26] propose generation of a population vector from which an elite solution is obtained. At each step, some solutions are sampled on the basis of selected mutation scheme. Halder et al [27] propose a cluster-based differential evolution algorithm for optimization in search environment. Elsayed et al [28] proposed an improved self-adaptive DE algorithm for optimization problem.

\section{Selection of the most relevant project using differential evolution algorithm}

The main advantage of DE is its ability to provide multiple solutions. It can be easily applied to real problems despite noisy and multidimensional space. It is simple but has an effective mutation process that ensures search diversity. Here, we propose a new model called DEAPS (Differential Evolution in Analogy for Project Selection). The following figure gives the framework for using differential evolution algorithm to select the relevant project from the available set of historical projects (figure 2).

The proposed method combines Analogy concept with differential evolution algorithm, The retrieval of the most similar project is done in two stages. In the first stage, there is a reduction of historical database to a set of most similar projects using the similarity measure. In the second stage, $\mathrm{DE}$ is applied to retrieve the most relevant project.

\subsection{Experimental results}

The input is the project parameters from the Desharnais data set and Albrechdt data set whose values are slightly changed so as to find the relevant project with the nearest parameter values. It is found that, by using the DE Algorithm, the most relevant project is retrieved. The data set is tested with three types of DE namely Simple DE, Population-based DE and Compact DE. The results are given in the table for the Desharnais data set (table 1):

The graphical representation of experimental results is given in figure 3 .

Lesser value of MMRE indicates that the model is successful. Thus our research proves that by using the DEAPS model, it is possible to retrieve the most relevant project from the historical database in an efficient manner (figure 4).

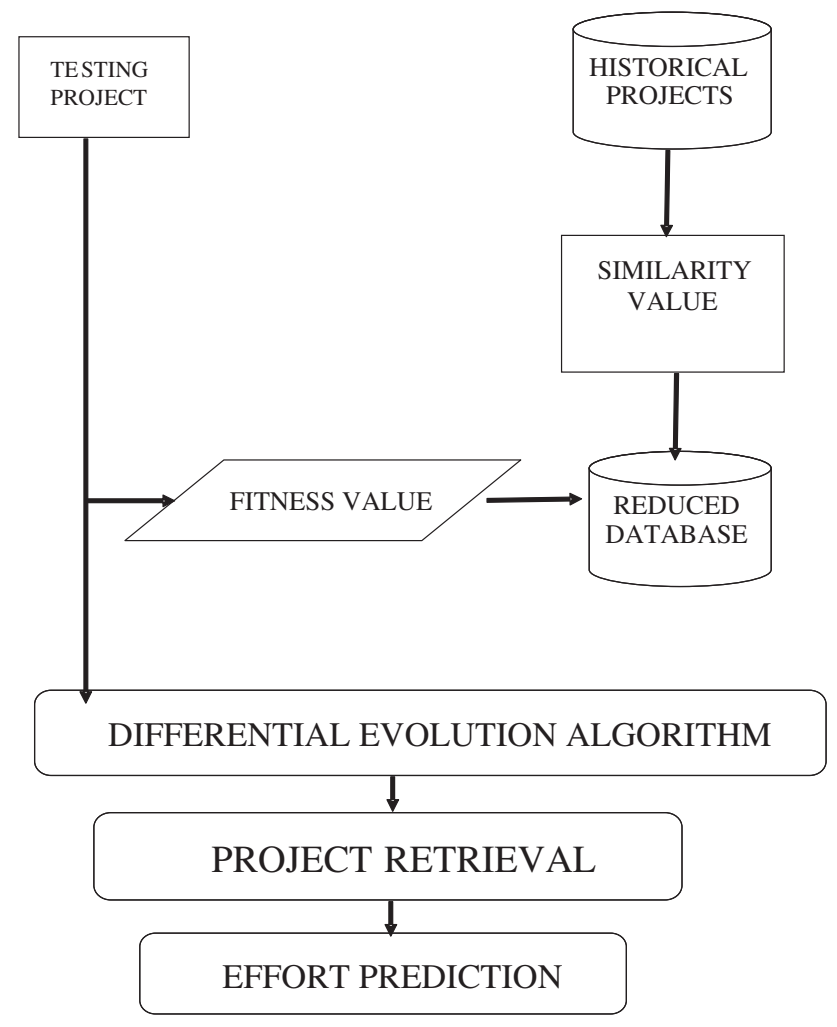

Figure 2. Differential evolution in the selection of relevant project. 
Table 1. Results of DEAPS model on Desharnais data set.

\begin{tabular}{lccc}
\hline & \multicolumn{3}{c}{ MRE values } \\
\cline { 2 - 4 } Project Id & Simple DE & Population-based DE & Compact DE \\
\hline 10 & 0.5644 & 0.6532 & 0.6532 \\
14 & 0.7970 & 0.8298 & 0.8298 \\
18 & 0.0917 & 0.0917 & 0.0917 \\
19 & 0.2904 & 0.2904 & 0.2904 \\
22 & 0.2617 & 0.2901 & 0.2617 \\
31 & 0.3583 & 0.3583 & 0.3583 \\
36 & 0.3052 & 0.3052 & 0.3052 \\
40 & 0.0712 & 0.0712 & 0.0712 \\
46 & 0.3933 & 0.3933 & 0.3933 \\
55 & 0.1401 & 0.1401 & 0.1401 \\
MMRE & 0.3273 & 0.3423 & 0.3395 \\
\hline
\end{tabular}

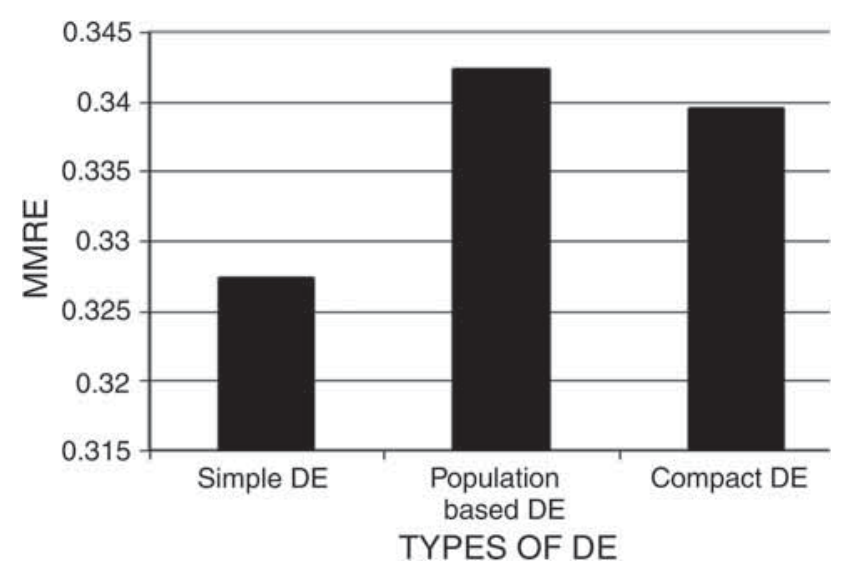

Figure 3. MMRE value comparison for different types of DE.

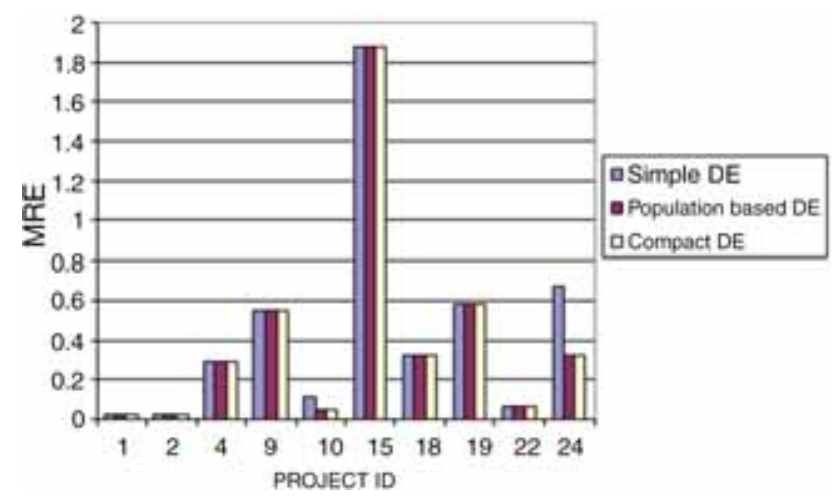

Figure 4. MRE value comparison for different types of DE.

\subsection{Data set}

To explore and compare the potential of the software effort estimation models, data sets are used. The most commonly used data sets are the Desharnais data set, Albrechdt data set and COCOMO 81 data set. Each data set consists of a different number of projects with different parameters. The
Table 2. Features of data sets.

\begin{tabular}{lccc}
\hline Sl.no. & Data set & No. of projects & No. of parameters \\
\hline 1 & Albrechdt & 24 & 7 \\
2 & Deshairnais & 77 & 11 \\
3 & COCOMO 81 & 63 & 16 \\
\hline
\end{tabular}

Desharnais data set consists of 81 software projects of a Canadian software house. Although it is old, it is one of the larger, publicly available data sets. The main reason for using these datasets is that they have been widely used to empirically validate a large amount of research result. Also they are publicly available (table 2).

The above-listed data sets are most popularly used by the researchers for the performance evaluation. However there are other data sets used in various research works. This includes the Maxwell data set, Kemerer data set, ISBSG (International Software Benching Standards Group), etc.

\subsection{Performance evaluation metrics}

Evaluation criteria are essential for the validation of Effort Estimation Models. Metrics are used for this purpose. The most commonly used metrics are given below.

a. Magnitude of relative error (MRE) Relative error is the difference between the actual value and estimated value. MRE is the absolute value of the relative error.

$$
\operatorname{MRE}=\frac{|A-E|}{|A|}
$$

where $A$ is the Actual Effort Value and $E$ is the Estimated Effort Value.

b. Mean magnitude of relative error (MMRE) MMRE is the average percentage of the MRE over an entire data set:

$$
\operatorname{MMRE}=\sum_{i=1}^{i=n}\left|\frac{A i-E i}{A i}\right| * \frac{100}{n}
$$

where $A i$ is the Actual Effort and $E i$ is the Estimated Effort of the $i$ th project; $n$ is the number of projects.

c. $\operatorname{Pred}(\mathrm{q})$ : The prediction level $\operatorname{pred}(q)$ is the average percentage of prediction that falls within a specified percentage $(q \%)$ of the actual value. If the value of $\operatorname{pred}(q)$ is high, then the estimation is good:

$$
\operatorname{pred}(q)=\frac{p}{n}
$$

where $p$ is the number of projects whose MRE is less than or equal to $q$. The commonly used metric is $\operatorname{pred}(.25)$, which is the percentage of predictions that is less than $25 \%$ of the actual value:

$$
\operatorname{pred}(.25)=\frac{1}{n} \sum_{i=1}^{n}\left(\frac{|A i-E i|}{|A i|}\right) \leq 0.25 .
$$


d. Balanced mean magnitude of relative error (BMMRE)

$$
\mathrm{BMMRE}=\sum_{i=1}^{n}\left(\frac{|A i-E i|}{\min (A i, E i)}\right) \frac{100}{n} .
$$

e. Root Mean Square (RMS): RMS is the square root of the mean of the square of the difference between the actual and estimated effort.

$$
\mathrm{RMS}=\sqrt{\frac{1}{n} \sum_{i=1}^{n}(A i-E i)^{2}} .
$$

\section{Conclusion and future work}

The aim of this paper is to provide a systematic review of papers on software development effort estimation and to propose a new model to select the relevant project in analogy. The main reason for the failure of software projects is inaccurate effort estimation. There are so many models available for software effort estimation but there is no single particular model that can suite all type of projects. The performance of each estimation method depends on several parameters such as size, project duration, complexity of the project, expertise of the staff and development method. The evaluation metric also plays an important role in deciding the right estimation model. According to our research, the most commonly used metric for evaluating the software estimation model is MMRE.

The evolutionary algorithms such as GA and DE are promising methodologies in the field of software effort estimation. The model DEAPS that is proposed in this paper is based on the differential evolution algorithm. This model can be used in cases where the software projects are described by categorical or numeric data. The experimental results of checking the model on the Desharnais data set are given in this paper. Future work is to test the model DEAPS with a few more data sets and prove that this is a very efficient method to retrieve the most relevant project from historical databases.

\section{References}

[1] Jorgensen M, Grusehke T M and Gupta R 2009 The impact of lessons - learned sessions on effort estimation and uncertainity assessments. IEEE Trans. Softw. Eng. 35(3): 368-383

[2] Nan N and Harter D E 2009 Impact of budget and schedule pressure on software development cycle time and effort. IEEE Trans. Softw. Eng. 35(5): 624-637

[3] Laird L M 2006 The limitations of estimation. IT Prof. 8(6): 40-45
[4] Jorgensen M and Sheppard M 2007 A systematic review of software development cost estimation studies. IEEE Trans. Softw. Eng. 33(1): 33-53

[5] Dejaeger K, Verbeke W, Martens D and Baesens B 2012 Data mining techniques for software effort estimation: a comparative study. IEEE Trans. Softw. Eng. 38: 375-397

[6] Menzies T, Butcher A, Cok D, Layman L, Shull F and Turhan B 2013 Local vs global lessons for defect prediction and effort estimation. IEEE Trans. Softw. Eng. 39: 822-834

[7] Kocaguneli E, Menzies T, Bener A B and Keung J W 2012 Exploiting the essential assumptions of analogy based effort estimation. IEEE Trans. Softw. Eng. 38(2): 425-437

[8] Mittas N and Angelis L 2013 Ranking and clustering software cost estimation model through a multiple comparison algorithm. IEEE Trans. Softw. Eng. 39(4): 537-551

[9] Harman M and Mausouri A 2010 Search based software engineering: introduction to special issue of the IEEE Transactions on Software Engineering. IEEE Trans. Softw. Eng. 36(6): 737-741

[10] Kocaguneli E, Menzies T, Keung J, Cok D and Madachy R 2013 Active learning and effort estimation: finding the essential content of software effort estimation data. IEEE Trans. Softw. Eng. 39(8): 1040-1053

[11] Ashman R 2004 Project estimation: a simple use-case-based model. IT Prof. 6(4): 40-44

[12] Jorgensen M 2005, Evidence based guidelines for assessment of software development cost uncertainty. IEEE Trans. Softw. Eng. 31(11): 942-954

[13] Hsu C J, Rodas N U, Huang C Y and Peng K L 2010 A study of improving the accuracy of software effort estimation using linear weighted combination. In: Proceedings of the Annual IEEE Computer Software Applications Conference, pp. $98-103$

[14] Jorgensen M and Grimstad S 2011 The impact of irrelevant and misleading information on software development effort estimates: a randomized controlled field experiment. IEEE Trans. Softw. Eng. 37(5): 695-707

[15] Jørgensen M 2004 A review of studies on expert estimation of software development effort. J. Syst. Softw. 70(1-2): $37-60$

[16] Menzies T, Chen Z, Hihn J and Lum K 2006 Selecting best practices for effort estimation. IEEE Trans. Softw. Eng. 32(11): 883-895

[17] Auer M, Trendowicz A, Graser B, Haunschmid E and Biffl S 2006 Optimal project feature weights in analogy-based cost estimation: improvement and limitations. IEEE Trans. Softw. Eng. 32(2): 83-92

[18] Suwanjang H and Prompoon N 2012 Framework for developing a software cost estimation model for software modification based on a relational matrix of project profile and software cost using an analogy. Int. J. Comput. Commun. Eng. 1: 129-134

[19] Mair C, Kadoda G, Lefley M, Phalp K, Schofield C, Shepperd S and Webster S 2000 An investigation of machine learning based prediction systems. J. Softw. Syst. 53(1): 23-29

[20] Finnie G R and Wittig G E 1996 AI tools for software development effort estimation. In: Proceedings of the International Conference on Software Engineering Education and Practice, pp. 83-92 
[21] Malhotra R and Jain A 2011 Software effort prediction using statistical and machine learning methods. Int. J. Adv. Comput. Sci. Appl. 2(1): 145-152

[22] Li Y F, Xie M and Goh T N 2010 A study of genetic algorithm for project selection for analogy based software cost estimation. In: Proceedings of IEEE IEEM, pp. 1256-1260

[23] Krogmaun K, Kuperberg M and Reussner R 2007 Using genetic search for reverse engineering of parametric behavior models for performance prediction. IEEE Trans. Softw. Eng. 36(6): 865-877

[24] Burgess C J and Lefley M 2001 Can genetic programming improve software effort estimation? A comparative evaluation. Inf. Softw. Technol. 43(14): 863-873
[25] Thamarai I and Murugavalli S 2012 Using differential evolution in the prediction of software effort. In: Proceedings of the Fourth International Conference on Advanced Computing, pp. 1-3

[26] Mininno E, Neri F, Cupertino F and Naso D 2011 Compact differential evolution. IEEE Trans. Evol. Comput. 15(1): 32-53

[27] Halder U, Das S and Naity D 2013 A cluster based Differential Evolution Algorithm with external archive for optimization in dynamic environments. IEEE Trans. Syst. Maint. Cybern. 43(3): 881-897

[28] Elsayed S M, Sarker R A and Essam D L 2013 An improved self-adaptive Differential Evolution Algorithm for optimization problems. IEEE Trans. Ind. Informat. 9(1): 89-99 